\title{
Superoxide dismutase promotes the epithelial-mesenchymal transition of pancreatic cancer cells via activation of the $\mathrm{H}_{2} \mathrm{O}_{2} / \mathrm{ERK} / \mathrm{NF}-\kappa \mathrm{B}$ axis
}

\author{
WEI LI $^{1 *}$, LEI CAO ${ }^{2 *}$, LIANG HAN $^{1}$, QINHONG XU $^{1}$ and QINGYONG MA ${ }^{1}$ \\ ${ }^{1}$ Department of Hepatobiliary Surgery, First Affiliated Hospital of Medical College, ${ }^{2}$ Department of Pharmacology, \\ College of Medicine, Xi'an Jiaotong University, Xi'an 710061, P.R. China
}

Received January 17, 2015; Accepted March 3, 2015

DOI: $10.3892 /$ ijo.2015.2938

\begin{abstract}
Our previous study revealed that superoxide dismutase (SOD)-dependent production of reactive oxygen species (ROS) was able to increase the invasive ability of pancreatic cancer cells. However, the underlying mechanisms by which SOD enhances metastasis are still not fully elucidated. As epithelial-mesenchymal transition (EMT) is a key player in tumor metastasis, the aim of this study was to evaluate whether SOD affects EMT in pancreatic cancer cells and the related mechanism. Human pancreatic cancer cells BxPC-3 and Panc-1 were utilized to examine the level of hydrogen peroxide $\left(\mathrm{H}_{2} \mathrm{O}\right)$ in the absence or presence of SOD and catalase (CAT). The activation of phospho-ERK and phospho-NF- $\mathrm{KB}$ were measured by western blot analysis. Wound healing assay and transwell invasion assay were used to detect the migratory and invasive potential of cancer cells. The EMT-related factors, E-cadherin, $\mathrm{N}$-cadherin and vimentin were detected by QT-PCR and western blot analysis. The results of present study showed that SOD not only increased cell migration and invasion in pancreatic cancer, but also mediated the expression of EMT-related factors and cell morphology. In addition, the levels of phospho-ERK and phospho-NF- $\kappa \mathrm{B}$ were induced by SOD which could be counter-balanced by both CAT treatment and PD 98059 (an ERK inhibitor). Taken together, these data indicate that SOD promotes the invasive and migratory activity of pancreatic cancer. Blocking the $\mathrm{H}_{2} \mathrm{O}_{2} / E R K / N F-\kappa B$ axis might be a novel strategy for the treatment of this severe malignancy.
\end{abstract}

Correspondence to: Dr Qingyong Ma, Department of Hepatobiliary Surgery, First Affiliated Hospital of Xi'an Jiaotong University, 277 West Yanta Road, Xi'an 710061, P.R. China

E-mail: qyma56@mail.xjtu.edu.cn

${ }^{*}$ Contributed equally

Key words: superoxide dismutase, hydrogen peroxide, epithelialmesenchymal transition, ERK pathway, pancreatic cancer

\section{Introduction}

As the fourth leading cause of cancer death worldwide, pancreatic cancer is an aggressive malignant disease with a median survival time of less than 6 months and a 5-year survival rate of $<6 \%$ (1). Due to a lack of early symptoms, $\sim 80 \%$ of pancreatic cancer patients are advanced in unresectable stage at the time of diagnosis (2). Even patients with seemingly resectable tumor are not always cured by surgery due to the microscopic systemic spread of the pancreatic cancer that occurs prior to the procedure (3). Improvement of patient survival requires an increased understanding of tumor metastasis mechanisms to allow for early disease detection and the development of therapeutic strategy. Previously we identified that superoxide dismutase (SOD)-dependent production of reactive oxygen species (ROS) increased the invasive and migratory ability of pancreatic cancer cells (4). However, little is known about the specific mechanism underlying this linkage.

ROS generated by the mitochondrial respiratory chain, consists of a number of chemically reactive molecules derived from oxygen, including hydrogen peroxide $\left(\mathrm{H}_{2} \mathrm{O}_{2}\right)$. Accumulating evidence indicates that the intracellular redox state plays an important role in cellular signaling transduction and regulates multiple events, such as cell cycle progression, apoptosis, migration, invasion and angiogenesis in cancer $(5,6)$. The main antioxidant enzymes include SOD, which converts superoxide anion $\left(\mathrm{O}_{2}^{-}\right)$into $\mathrm{H}_{2} \mathrm{O}_{2}$, as well as catalase (CAT) and glutathione peroxidase (GPX), which catabolize $\mathrm{H}_{2} \mathrm{O}_{2}$ into water (7). Although they are sometimes classified together, they should be considered separately because their reactions are completely different. It has been demonstrated that SOD can induce migration and invasion of cancer cells via its metabolic product $\mathrm{H}_{2} \mathrm{O}_{2}$. Whereas, the $\mathrm{H}_{2} \mathrm{O}_{2}$ scavenger, catalase inhibits ROS-mediated tumor metastasis $(4,8)$. In pancreatic cancer, the antioxidant activity of SOD is increased in the tumor cells cultured from ascites and metastatic tumor cell lines compared to primary pancreatic tumor cells, which indicates that SOD is intimately related with tumor metastasis potential (9).

Epithelial-mesenchymal transition (EMT) has been recognized not only as a physiological mechanism for development 
and tissue remodeling, but also as a pathological mechanism in cancer progression, during which cells lose their polarized epithelial traits and acquire mesenchymal characteristics such as the downregulation of E-cadherin and the upregulation of $\mathrm{N}$-cadherin and vimentin that in turn induce an aggressive phenotype $(10,11)$. This event not only facilitates the aggressiveness of the disease but also promotes resistance to current treatments (12). Elevated amounts of ROS have been proven to promote several cellular migratory processes, including EMT, angiogenesis and metastasis in different cancer types (13). Various stimulators such as epidermal growth factor and transforming growth factor- $\beta$ could also induce EMT through ROS generation (14). As one of the most classical signaling pathway, extracellular signal-regulated kinase (ERK) pathway is an important signaling cascade downstream of ROS that is involved in tumor migration and invasion (15).

In the present study, we investigated the production of $\mathrm{H}_{2} \mathrm{O}_{2}$ in pancreatic cancer cells in response to SOD and CAT. We also tested the hypothesis that $\mathrm{H}_{2} \mathrm{O}_{2}$ mediates SOD-induced activation of ERK signaling pathway, which further regulates the invasive and migratory activity of pancreatic cancer cells through EMT. Results from this study suggest that elevation of $\mathrm{H}_{2} \mathrm{O}_{2}$ contributes to the SOD induced EMT through ERK/ $\mathrm{NF}-\kappa \mathrm{B}$ signaling pathway.

\section{Materials and methods}

Preparation of chemicals. Dulbecco's modified Eagle's medium (DMEM) and fetal bovine serum (FBS) were from Gibco (Grand Island, NY, USA). SOD and CAT were from Sigma-Aldrich (St. Louis, MO, USA). The hydrogen peroxide assay kit was from Beyotime (Jinan, China). Millicell culture plate inserts were from Millipore (Bedford, MA, USA). Matrigel was from BD (Biosciences, Bedford, MA, USA). The ERK inhibitor PD 98059 was obtained from SigmaAldrich. Primary antibodies against E-cadherin, N-cadherin and vimentin were from Santa Cruz Biotechnology (Santa Cruz, CA, USA). The anti-ERK, anti-phospho-ERK (Thr202/

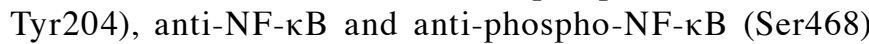
antibodies were from Cell Signaling Technology (Beverly, MA, USA). Nitrocellulose membranes were from Millipore. The BCA assay kit and the chemiluminescence kit were from Pierce (Rockford, IL, USA). Other reagents were purchased from common commercial sources. All drug solutions were freshly prepared on the day of testing.

Cell cultures and treatments. The human pancreatic cancer cell lines, BxPC-3 and Panc-1, were obtained from the American Type Culture Collection (Manassas, VA, USA). The cells were cultured in DMEM medium containing $10 \%$ dialyzed heat-inactivated FBS, $100 \mathrm{U} / \mathrm{ml}$ penicillin, and $100 \mu \mathrm{g} / \mathrm{ml}$ streptomycin in a humidified atmosphere of $5 \%$ $\mathrm{CO}_{2}$ at $37^{\circ} \mathrm{C}$. SOD $(400 \mathrm{U} / \mathrm{ml})$ was used to convert superoxide anions into molecular oxygen and hydrogen peroxide, CAT (400 U/ml) was also added to the in vitro model in combination with SOD to decompose the hydrogen peroxide.

Hydrogen peroxide assay. The level of intracellular $\mathrm{H}_{2} \mathrm{O}_{2}$ was measured using hydrogen peroxide assay kit according to the manufacturer's instructions. In this kit, the ferrous ions $\left(\mathrm{Fe}^{2+}\right)$ were oxidized to ferric ions $\left(\mathrm{Fe}^{3+}\right)$ by $\mathrm{H}_{2} \mathrm{O}_{2}$, which further formed a complex with the indicator dye xylenol orange and produced a visible purple-colored complex, that could then be measured using a microplate reader at a wavelength of 560-590 nm (Bio-Rad, CA, USA).

Transwell Matrigel invasion assay. The invasive ability of the pancreatic cancer cells was analyzed using Matrigel invasion chambers in 24-well plates. The $8.0-\mu \mathrm{m}$ pore inserts were coated with $25 \mu \mathrm{l}$ of Matrigel. After serum starvation for $24 \mathrm{~h}$, the BxPC-3 and Panc- 1 cells were suspended in DMEM containing $1 \%$ FBS in the top chamber at a concentration of $5 \times 10^{4}$ in the absence or presence of SOD, SOD accompanied with CAT at concentrations of $400 \mathrm{U} / \mathrm{ml}$ each and SOD accompanied with PD 98059 (50 $\mu \mathrm{mol} / \mathrm{l})$. Simultaneously, $500 \mathrm{ml}$ of DMEM containing 20\% FBS was placed in the lower chambers. The Matrigel invasion chamber was then incubated for $48 \mathrm{~h}$ in a humidified tissue culture incubator. The non-invading cells were removed from the upper surface by scraping with a wet cotton swab. After rinsing with PBS, the filter was fixed and stained with crystal violet. The invasion ability was determined by counting the stained cells on the bottom surface. Three random fields were captured at $\times 20$ magnification $(n=3)$.

Wound healing assay. Cell migratory ability was detected by a wound-healing assay. Pancreatic cancer cells were seeded in 24 -well plates $\left(1.0 \times 10^{5}\right.$ cells $\left./ 500 \mu \mathrm{l}\right)$. After the cells grew to $90-100 \%$ confluence, a sterile pipette tip was used to produce a wound line between the cells. Cellular debris was removed by washing with PBS and then allowed to migrate for $24 \mathrm{~h}$. Images were taken at time 0 and $24 \mathrm{~h}$ post-wounding under a Nikon Diaphot TMD inverted microscope (x10). The relative distance traveled by the leading edge from 0 to $24 \mathrm{~h}$ was assessed using Photoshop software $(n=5)$.

Real-time quantitative PCR (QT-PCR). Total RNA was extracted from the pancreatic cancer cells using the Fastgen 200 RNA isolation system (Fastgen, Shanghai, China) according to the manufacturer's protocol. Total RNA was reverse-transcribed into cDNA using the Fermentas RevertAid ${ }^{\mathrm{TM}}$ kit (MBI Fermentas, Canada). The primer sequences were as follows: E-cadherin-F, 5'-ATTCTGATTCTGCTGCTCTTG-3' and E-cadherin-R, 5'-AGTCCTGGTCCTCTTCTCC-3'; N-cadherin-F, 5'-TGTTTGACTATGAAGGCAGTGG-3' and $\mathrm{N}$-cadherin-R, 5'-TCAGTCATCACCTCCACCAT-3'; vimentin-F, 5'-AATGACCGCTTCGCCAAC-3' and vimentin-R, 5'-CCG CATCTCCTCCTCGTAG-3'; $\beta$-actin-F, 5'-GACTTAGTT GCGTTACACCCTTTCT-3' and $\beta$-actin-R, 5'-GAACGGT GAAGGTGACAGCAGT-3'. The PCR reactions consisted of $30 \mathrm{sec}$ at $95^{\circ} \mathrm{C}$, followed by 40 cycles of $95^{\circ} \mathrm{C}$ for $5 \mathrm{sec}, 60^{\circ} \mathrm{C}$ for $30 \mathrm{sec}$ and $72^{\circ} \mathrm{C}$ for $30 \mathrm{sec}$. After each QT-PCR experiment, a dissociation curve analysis was conducted. The relative gene expression was calculated using the previously described $2^{-\Delta \Delta \mathrm{Ct}}$ method (16).

Protein extraction and western blotting. Total protein was extracted from cultured cells in radio-immunoprecipitation assay (RIPA) lysis buffer on ice for $25 \mathrm{~min}$. Insoluble materials were removed by centrifugation at $4^{\circ} \mathrm{C}$ with $15,000 \mathrm{x} \mathrm{g}$ for $15 \mathrm{~min}$. Subsequently, supernatants were collected and 


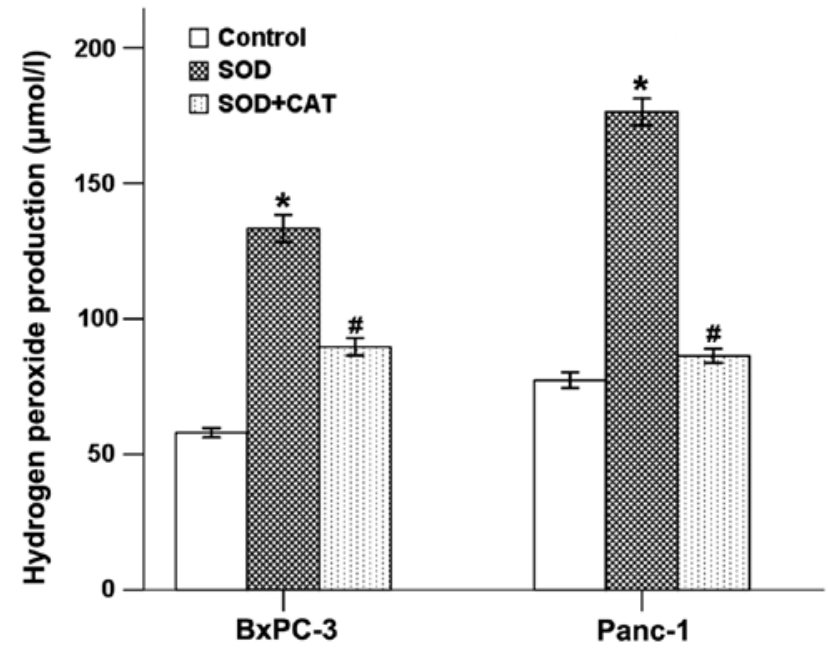

Figure 1. SOD promotes the production of hydrogen peroxide in pancreatic cancer cells. After BxPC-3 and Panc-1 cells were treated with SOD in the absence or presence of CAT for $24 \mathrm{~h}, \mathrm{H}_{2} \mathrm{O}_{2}$ levels were measured using a hydrogen peroxide assay kit. ${ }^{*} \mathrm{P}<0.05$ as compared with control group; ${ }^{\#} \mathrm{P}<0.05$ as compared with SOD group.

total protein concentrations were measured using the BCA assay kit. Proteins (30-80 $\mu \mathrm{g}$ ) were electrophoretically resolved on a denaturing SDS-polyacrylamide gel and electrotransferred onto nitrocellulose membranes. The membranes were initially blocked with $5 \%$ non-fat dry milk in Tris-buffered saline (TBS) for $2 \mathrm{~h}$ and then probed with antibodies against E-cadherin, $\mathrm{N}$-cadherin, vimentin, ERK, phospho-ERK, NF- $\kappa$ B, phospho-NF- $\kappa$ B and $\beta$-actin. After incubation with the primary antibodies at $4^{\circ} \mathrm{C}$ overnight, the membranes were hybridized with secondary goat antimouse or goat anti-rabbit antibodies (Sigma-Aldrich) for $2 \mathrm{~h}$ at room temperature. Immunopositive bands were developed using an enhanced chemiluminescence (ECL) detection system (Amersham, Piscataway, NJ, USA). All analyses were conducted in triplicate.

Statistical analysis. Statistical analysis was performed using SPSS software (version 17.0, SPSS Inc., Chicago, IL, USA). Data are presented as the means \pm SEM of three replicate assays. Differences between the groups were analyzed by analysis of variance (ANOVA). Statistical significance was set at $\mathrm{P}<0.05$. All experiments were repeated independently at least three times.

\section{Results}

SOD increases the production of $\mathrm{H}_{2} \mathrm{O}_{2}$ in pancreatic cancer cells. The intracellular $\mathrm{H}_{2} \mathrm{O}_{2}$ in BxPC-3 and Panc- 1 cells treated with SOD or SOD accompanied with CAT was determined using a hydrogen peroxide assay kit detected by a microplate reader. As shown in Fig. 1, the levels of $\mathrm{H}_{2} \mathrm{O}_{2}$ in both the BxPC-3 and Panc-1 cells were upregulated in response to SOD stimulation. CAT could counter-balance the effect of SOD in both cancer cell types.

SOD upregulates the activation of the ERK/NF- $\kappa B$ signaling pathway. As an important member of mitogen activated
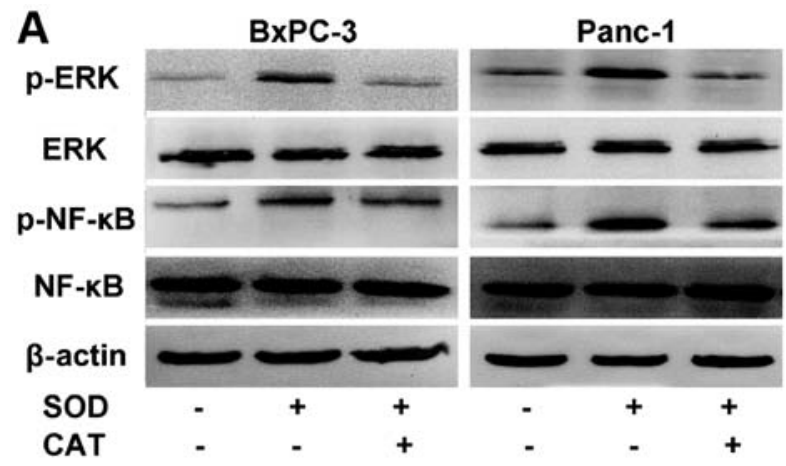

B

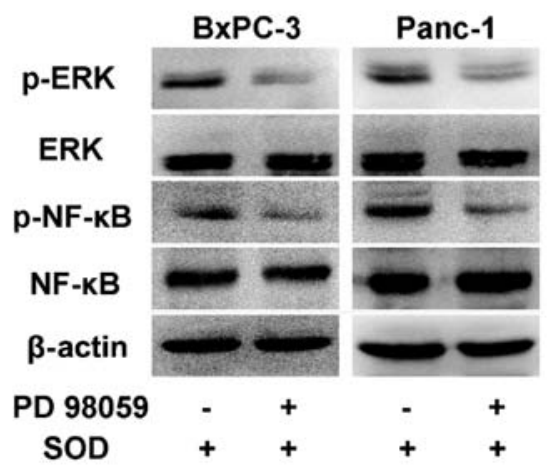

Figure 2. The roles of SOD, CAT and PD 98059 in the phosphorylation of ERK and NF- $\kappa$ B. (A) SOD promotes ERK and NF- $\kappa$ B phosphorylation that was counter-balanced by CAT. BxPC-3 and Panc-1 cells were exposed to $\mathrm{SOD}$ in the absence or presence of CAT for $24 \mathrm{~h}$ and whole cell lysates were prepared for western blotting. (B) Pancreatic cancer cells were treated with PD $98059(50 \mu \mathrm{mol} / \mathrm{l})$, an ERK inhibitor, for $24 \mathrm{~h}$ and used for western blotting to assess the activation of ERK and NF-кB.

protein kinase (MAPK) signaling pathway, ERK pathway is downstream of ROS which is involved in tumor migration and invasion (17). A recent study demonstrated that ERK pathway induces activation of NF- $\kappa \mathrm{B}$ transcription factor, and is associated with cell migration activity (18).

In the present study, we observed that both ERK and $\mathrm{NF}-\kappa \mathrm{B}$ phosphorylation were strongly increased with the addition of SOD (Fig. 2A). When we added SOD with CAT together into the cell culture, the expression of phospho-ERK and phospho-NF- $\mathrm{B}$ decreased significantly, indicating that the activation of ERK/NF- $\kappa \mathrm{B}$ signaling pathway in BxPC-3 and Panc-1 cells was hydrogen peroxide-dependent. In addition the ERK inhibitor PD 98059 was able to inhibit the expression of phospho-ERK and phospho-NF- $\kappa \mathrm{B}$, indicating that the NF- $\kappa \mathrm{B}$ transcription factor is modulated by the ERK pathway (Fig. 2B).

SOD promotes wound closure via the activation of $\mathrm{H}_{2} \mathrm{O}_{2} /$ ERK axis. Migration and invasion are two important aspects that lead to the ability of cancer cells to form metastases. In order to further characterize the role of SOD in promoting metastasis, we investigated the role of SOD in migration using a classic wound healing assay. Results showed that SOD caused a significant increase in the migration of both BxPC-3 and Panc-1 cells after incubation for $24 \mathrm{~h}$. Both CAT and PD 98059 suppressed these effects which proved that $\mathrm{H}_{2} \mathrm{O}_{2} /$ ERK axis mediated SOD-induced cell migratory ability (Fig. 3). 

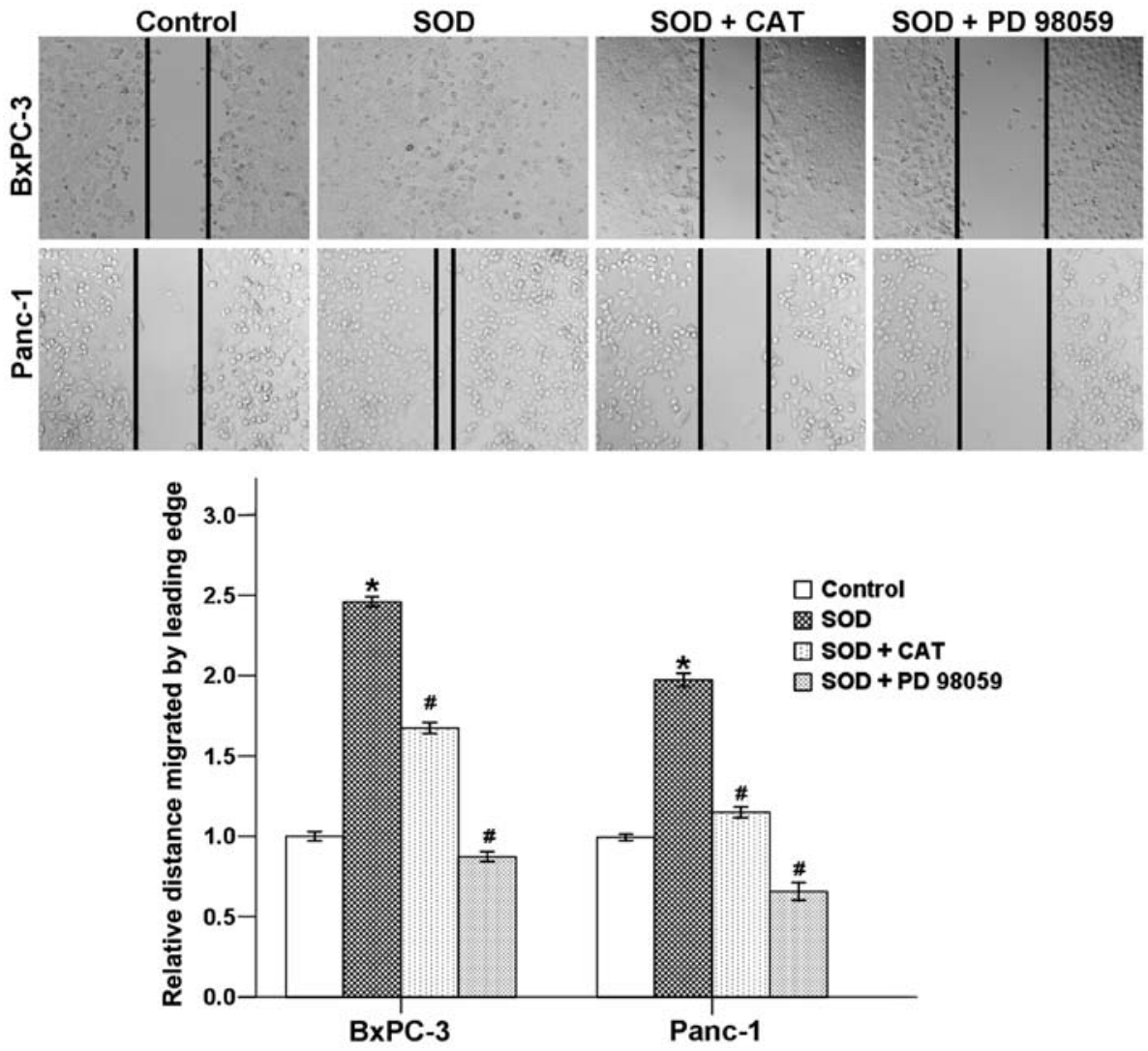

Figure 3. SOD promotes wound closure via the production of $\mathrm{H}_{2} \mathrm{O}_{2}$ and ERK signaling. The confluent monolayer was wounded with sterile pipette tip and cells were allowed to migrate for $24 \mathrm{~h}$. The migratory ability of BxPC-3 and Panc-1 cells were promoted by SOD. PD 98059 exposure as well as CAT addition for $24 \mathrm{~h}$ reduced the migration of pancreatic cancer cells. ${ }^{*} \mathrm{P}<0.05$ as compared with control group; ${ }^{*} \mathrm{P}<0.05$ as compared with SOD group.

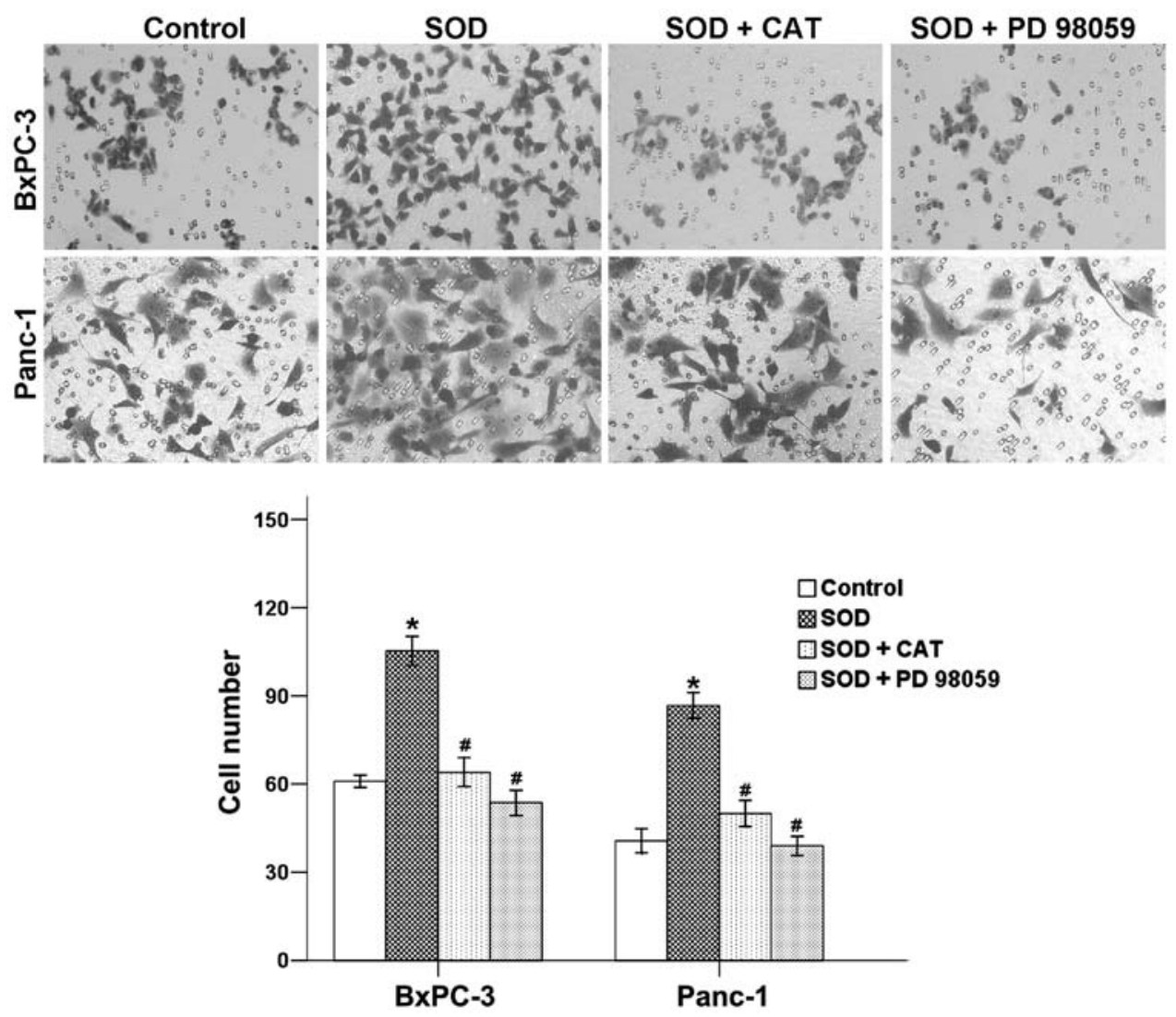

Figure 4. The effects of ERK signaling on SOD-induced invasion of pancreatic cancer cells. The images show the bottom side of the filter inserts with stained cells that have migrated through the filter pores after $48 \mathrm{~h}$. The invasive ability of both BxPC-3 and Panc-1 cells were promoted by the SOD, whereas, PD 98059 as well as CAT addition reduced the invasion of pancreatic cancer cells. ${ }^{*} \mathrm{P}<0.05$ as compared with control group; ${ }^{*} \mathrm{P}<0.05$ as compared with SOD group. 
A

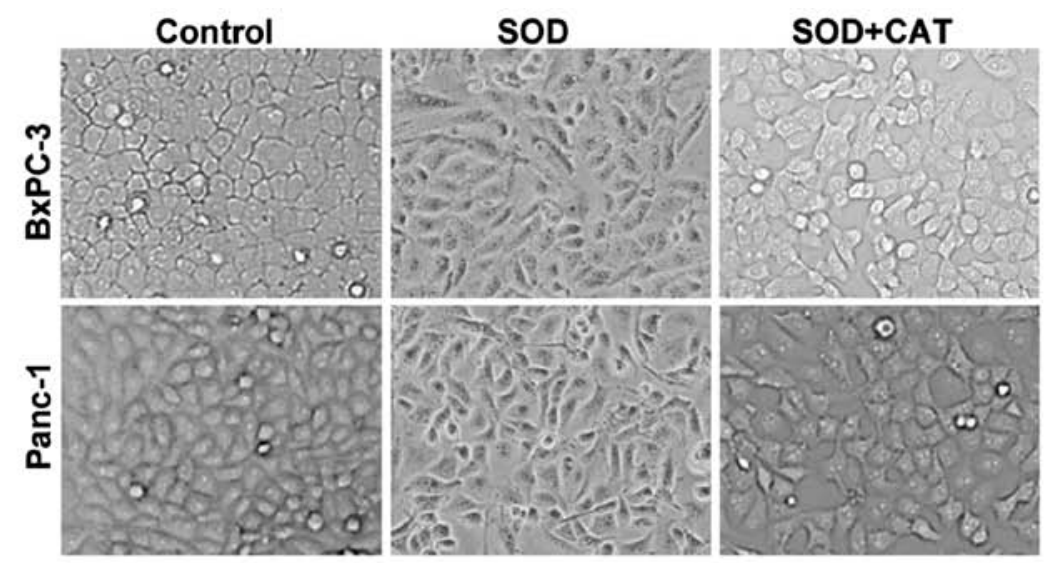

B

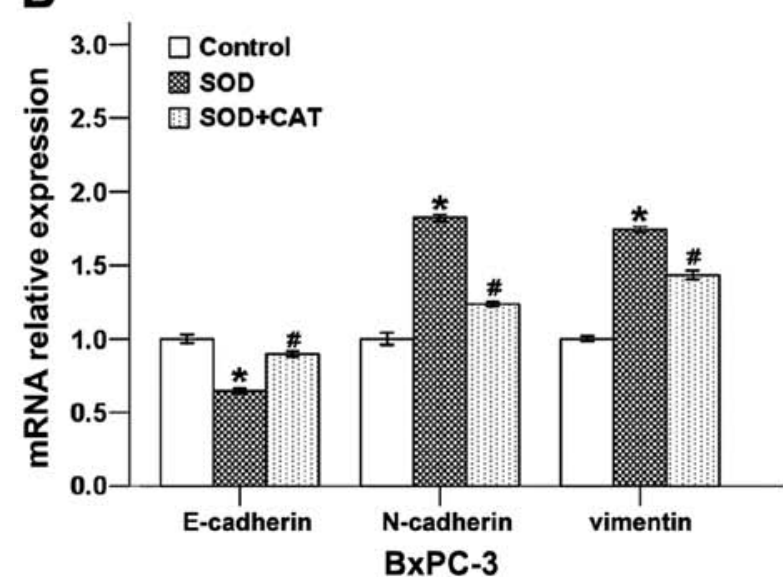

C

BxPC-3
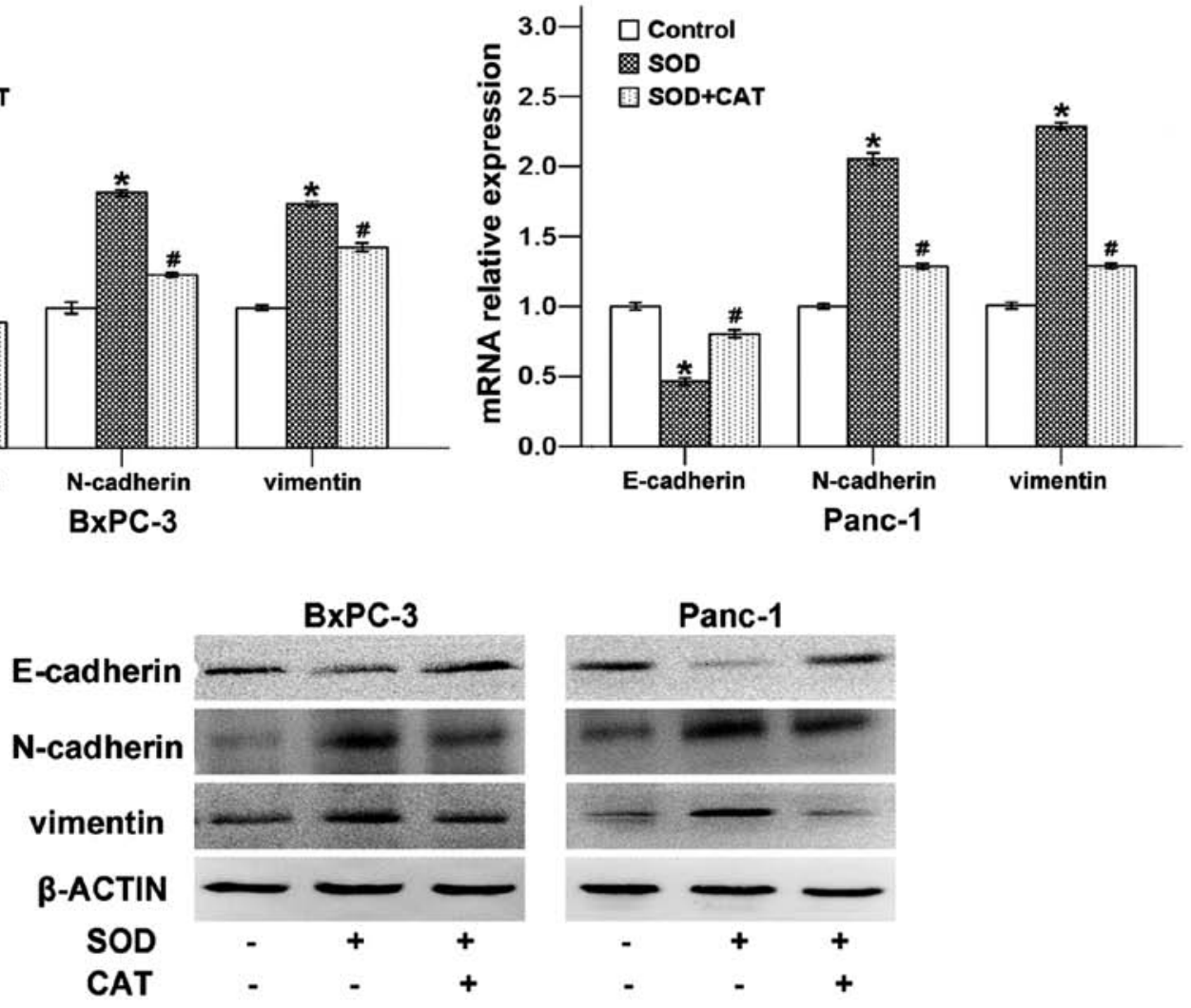

Panc-1

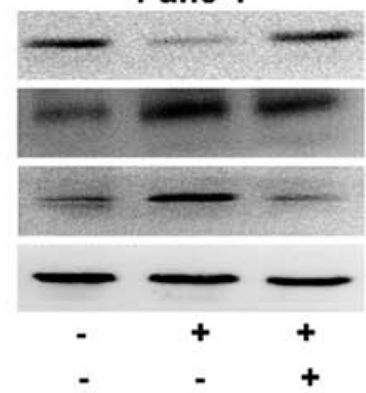

Figure 5. The effects of $\mathrm{H}_{2} \mathrm{O}_{2}$ on SOD-induced EMT of pancreatic cancer cells. (A) Cells were treated with SOD for $48 \mathrm{~h}$ and the phenomenon of EMT was observed based on morphological changes of the cells. (B) Treatment with CAT diminished the effects of SOD on the expression of E-cadherin, N-cadherin and vimentin at the mRNA level in BxPC-3 and Panc-1 cells, as determined by QT-PCR. (C) Treatment with CAT also diminished the effects of SOD on the expression of EMT-related genes at the protein level in both cancer cells, as determined by western blotting. ${ }^{*} \mathrm{P}<0.05$ as compared with control group; ${ }^{\#} \mathrm{P}<0.05$ as compared with SOD group.

Activation of $\mathrm{H}_{2} \mathrm{O}_{2} /$ ERK axis is responsible for the promotion of cancer cell invasion. To gain more insight into the role of $\mathrm{H}_{2} \mathrm{O}_{2} /$ ERK axis in cancer progression, BxPC-3 and Panc-1 cells were treated with SOD in the absence or presence of CAT and PD 98059. As shown in Fig. 4, the average cell numbers that invaded into the lower chamber increased with the addition of SOD after incubation for $48 \mathrm{~h}$. This increase was reversed by co-treating with both CAT and PD 98059. These finding revealed that $\mathrm{H}_{2} \mathrm{O}_{2} / \mathrm{ERK}$ axis might be an effective inhibitor of the migration and invasion of pancreatic cancer cells.
SOD induces EMT via $\mathrm{H}_{2} \mathrm{O}_{2}$ production in pancreatic cancer cells. EMT, a critical character of tumor metastasis, contains three essential processes: first, alterations of cell-cell and cell-extracellular matrix (ECM) interactions occur releasing the epithelial cells from the surrounding tissue. Then the cytoskeleton is reorganized so that the cells can gain the ability to move through ECM. After that, a new transcriptional program is induced to acquire morphological and functional characteristics of mesenchymal-like cells (6). In our study, we demonstrated that after treated with SOD for $48 \mathrm{~h}$, the cellular morphology of both BxPC-3 and Panc- 1 cells was changed 
from an epithelial phenotype to a classical mesenchymal phenotype. Cells treated with SOD and CAT displayed classical epithelial morphology (Fig. 5A).

To further confirm the effect of $\mathrm{H}_{2} \mathrm{O}_{2}$ on SOD-induced EMT, we determined the expression levels of EMT-related genes after the cells were SOD treated with or without CAT. As shown in Fig. 5B, SOD downregulated the mRNA level of the epithelial marker E-cadherin, while the expression of mesenchymal markers $\mathrm{N}$-cadherin and vimentin were strongly increased. CAT was able to significantly reverse these SOD-induced effects.

To evaluate the effects of SOD on the expression of E-cadherin, $\mathrm{N}$-cadherin and vimentin at protein level, we determined these proteins in BxPC-3 and Panc- 1 cells with or without CAT using western blotting. As shown in Fig. 5C, CAT counter-balanced the SOD-induced, EMT-related factors at the protein level, and the trend was consistent with the mRNA results. Taken together, our results demonstrate that SOD induces EMT progression via the production of $\mathrm{H}_{2} \mathrm{O}_{2}$ in both BxPC-3 and Panc-1 cells.

\section{Discussion}

As a fatal step in solid cancer progression, distant metastasis is responsible for approximately $90 \%$ of cancer-related deaths (19). It is commonly believed that the poor prognosis of pancreatic cancer is due to both the inherently aggressive biology of the disease and its late diagnosis in most cases (20). In recent years, EMT has received significant attention in cancer metastasis. Emerging evidence has suggested that EMT is the first step by which cancer cells invade and metastasize to other organs. Cancer cells undergoing EMT are able to obtain invasive properties to penetrate the surrounding tissue, leading to the creation of a suitable microenvironment for cancer proliferation and metastasis $(13,21)$. Our previous study has demonstrated that SOD-dependent production of ROS was able to increase the invasive and migratory ability of pancreatic cancer cells. In this study, we focus on the underlying mechanisms through which SOD promotes the metastasis ability in pancreatic cancer cell lines BxPC-3 and Panc-1.

Our data showed that SOD could significantly increase the production of intracellular $\mathrm{H}_{2} \mathrm{O}_{2}$ in BxPC- 3 and Panc- 1 cells, which further enhanced the capacity of the pancreatic cancer cells to migrate and invade the extracellular matrix. As the scavenger of $\mathrm{H}_{2} \mathrm{O}_{2}$, CAT was able to terminate these effects of SOD. ERK pathway has long been recognized as an important signaling cascade downstream of ROS that is involved in tumor migration and invasion (15). In order to determine whether the cell invasion and migration potential as well as the increased $\mathrm{H}_{2} \mathrm{O}_{2}$ production is related to ERK pathway, we tested the effects of SOD and CAT on the activation of phospho-ERK and the relative transcriptional factor NF- $\mathrm{\kappa B}$. With SOD alone, both ERK and NF- $\kappa$ B phosphorylation was strongly increased in pancreatic cancer cells, whereas the addition of CAT along with SOD to the cell culture resulted in a decrease of phospho-ERK and phospho-NF- $\kappa \mathrm{B}$. This phenomenon suggested that the activation of ERK/NF- $\mathrm{KB}$ pathway mediated by SOD was $\mathrm{H}_{2} \mathrm{O}_{2}$-dependent. In addition, the present study also showed that SOD was able to modulate cancer cell morphology as well as the expression of EMT-related factors, which could be counter-balanced by both CAT treatment and the ERK inhibitor PD 98059.

ROS, particularly, serve as regulators or secondary messengers of signal transduction pathways for cell proliferation, survival, apoptosis and migration (22). It has been proven that $\mathrm{H}_{2} \mathrm{O}_{2}$ is diffusible and thus capable of traveling across plasma membranes into the extracellular space to exert a paracrine role (23). Intrinsic antioxidant enzymes are vital to the regulation of oxidative stress within cells. SOD, one of the primary cellular antioxidants, catalyzes the conversion of superoxide anion to $\mathrm{H}_{2} \mathrm{O}_{2}$, which can be removed by CAT. Three forms of SOD exist in mammals that serve the same effect: copper-zinc SOD (CuZnSOD), manganese SOD (MnSOD) and extracellular SOD (EC-SOD). CuZnSOD is located in the cytoplasm, mitochondrial intermembrane space and the nucleus. MnSOD is located exclusively in the mitochondrial matrix, whereas EC-SOD is primary located in the extracellular matrix (ECM) (23).

Both epidemiologic and experimental evidence indicate that the levels of SOD increase in many tumor types as they progress from early stage non-invasive disease to late stage metastatic disease $(24,25)$. Increased SOD levels have been associated with poor prognosis and resistance to therapy of various tumors in the central nervous system, gastrointestinal tract, and head and neck (26). Toh et al (27) reported 2.19and 3.72-fold increases in SOD mRNA expression relative to normal tissue in gastric and colorectal cancers, respectively. Epidemiologic evidence has also linked a single nucleotide polymorphism in SOD, which increases its activity, to risk of developing cancers (28). The SOD2-1221G $>$ A AA genotype carriers had a significantly increased risk for pancreatic cancer among those with a low dietary vitamin E intake (29). Several authors have reported that SOD is involved in the invasive properties of cancer cells. It has been proven that metastatic pancreatic cancer cell line Capan-1, contain elevated levels of SOD and decreased levels of CAT, and that this change in steady-state levels of hydrogen peroxide correlates with increased metastasis, angiogenesis, proliferation and resistance to apoptosis $(9,24)$. Mice injected with MnSOD-GFP-transfected HT-1080 cells presented with multiple intrapulmonary metastatic lesions surrounding pulmonary blood vessels (30). Migration and invasion of the SOD-expressing HT-1080 fibrosarcoma and 253J transitional bladder carcinoma cells were inhibited following overexpression of CAT, indicating that the promigratory invasive phenotype of SOD-expressing cells is $\mathrm{H}_{2} \mathrm{O}_{2}$-dependent (30).

Increasing number of researchers have focused on the role of ROS in EMT-related cancer in recent years. Mori and colleagues first established a direct link between extracellular generation of ROS and EMT (31). In their study, normal mouse mammary gland epithelial cells were exposed to a low dose of $\mathrm{H}_{2} \mathrm{O}_{2}$ for periods of 2-4 days. A phenotypic conversion of mouse mammary epithelial cells from an epithelial to a fibroblast-like phenotype was observed, which was associated with the dissolution of cell-cell contacts, redistribution of E-cadherin in the cytoplasm, and upegulation of a set of integrin family members and matrix metalloproteinases (MMPs). Kim et al (32) reported that $\mathrm{H}_{2} \mathrm{O}_{2}$ could promote the EMT program, which was mediated through HIF-1 $\alpha$ and 
TGF- $\beta 1$ in human malignant mesothelioma (HMM) cells. Simultaneously, the $\mathrm{H}_{2} \mathrm{O}_{2}$ also increased the expression of stem cell-related genes, suggesting the enhanced potential of survival and proliferation of the HMM cells metastasis. Our results confirm that $\mathrm{SOD}$-induced $\mathrm{H}_{2} \mathrm{O}_{2}$ influences the migration and invasion via EMT in the pancreatic cancer cell lines BxPC-3 and Panc-1.

Members of the MAPK family include the extracellular ERK, c-jun NH-2 terminal kinase (JNK) and p38 MAPK. Numerous studies have proven the relationship between ERK signaling pathway and cancer progression. Lee et al (33) showed that hepatocyte growth factor (HGF) regulates $\mathrm{H}_{2} \mathrm{O}_{2}$ production, which further activates the ERK pathway and regulates uPA production, eventually increasing the invasive potential of stomach cancer cells. Liu et al (34) also demonstrated that bone morphogenetic protein 2-induced cellular invasiveness and MMP-2 activity is ROS/ERK-dependent in pancreatic cancer. Recent studies proved that the metastasismediated effect of the ERK signaling pathway might be modulated via the $\mathrm{NF}-\kappa \mathrm{B}$ transcription factor, which is associated with cell proliferation, cell migration and angiogenesis (35). Our results indicated that SOD was able to increase the activation of phospho-ERK and phospho-NF- $\kappa \mathrm{B}$ which was counter-balanced by CAT. After suppressing the ERK signaling pathway by PD 98059, the expression of phospho-ERK and phospho-NF- $\kappa \mathrm{B}$ as well as the invasion and migration ability of both $\mathrm{BxPC}-3$ and Panc- 1 pancreatic cancer cells were decreased.

In conclusion, the results of the present study suggest that SOD-induced $\mathrm{H}_{2} \mathrm{O}_{2}$ production can promote EMT in pancreatic cancer, leading to increased motility and invasion via activation of ERK signaling pathway. Managing $\mathrm{H}_{2} \mathrm{O}_{2} /$ ERK/ $\mathrm{NF}-\kappa \mathrm{B}$ axis might be a novel strategy for the treatment of this severe malignancy. Our findings warrant further investigation of this hypothesis.

\section{Acknowledgements}

This study was supported by grant from National Natural Science Foundation of China (grant no. 81301846).

\section{References}

1. Siegel R, Ma J, Zou Z and Jemal A: Cancer statistics, 2014. CA Cancer J Clin 64: 9-29, 2014

2. Sarkar FH, Banerjee S and Li Y: Pancreatic cancer: Pathogenesis, prevention and treatment. Toxicol Appl Pharmacol 224: 326-336, 2007.

3. Castellanos EH, Cardin DB and Berlin JD: Treatment of earlystage pancreatic cancer. Oncology (Williston Park) 25: 182-189, 2011.

4. Li W, Ma Q, Li J, Guo K, Liu H, Han L and Ma G: Hyperglycemia enhances the invasive and migratory activity of pancreatic cancer cells via hydrogen peroxide. Oncol Rep 25: 1279-1287, 2011.

5. Lee DJ and Kang SW: Reactive oxygen species and tumor metastasis. Mol Cells 35: 93-98, 2013.

6. Li W, Ma Q, Liu J, Han L, Ma G, Liu H, Shan T, Xie K and Wu E: Hyperglycemia as a mechanism of pancreatic cancer metastasis. Front Biosci (Landmark Ed) 17: 1761-1774, 2012.

7. Costa A, Scholer-Dahirel A and Mechta-Grigoriou F: The role of reactive oxygen species and metabolism on cancer cells and their microenvironment. Semin Cancer Biol 25: 23-32, 2014.

8. Liu Z, Li S, Cai Y, Wang A, He Q, Zheng C, Zhao T, Ding X and Zhou X: Manganese superoxide dismutase induces migration and invasion of tongue squamous cell carcinoma via $\mathrm{H}_{2} \mathrm{O}_{2}$-dependent Snail signaling. Free Radic Biol Med 53: 44-50, 2012.
9. Lewis A, Du J, Liu J, Ritchie JM, Oberley LW and Cullen JJ: Metastatic progression of pancreatic cancer: Changes in antioxidant enzymes and cell growth. Clin Exp Metastasis 22: 523-532, 2005.

10. Duan W, Li R, Ma J, Lei J, Xu Q, Jiang Z, Nan L, Li X, Wang Z, Huo $X$, et al: Overexpression of Nodal induces a metastatic phenotype in pancreatic cancer cells via the Smad2/3 pathway. Oncotarget 6: 1490-1506, 2015.

11. Inumaru J, Nagano O, Takahashi E, Ishimoto T, Nakamura S, Suzuki Y, Niwa S, Umezawa K, Tanihara $\mathrm{H}$ and Saya $\mathrm{H}$ : Molecular mechanisms regulating dissociation of cell-cell junction of epithelial cells by oxidative stress. Genes Cells 14: 703-716, 2009.

12. Barnett P, Arnold RS, Mezencev R, Chung LW, Zayzafoon M and Odero-Marah V: Snail-mediated regulation of reactive oxygen species in ARCaP human prostate cancer cells. Biochem Biophys Res Commun 404: 34-39, 2011.

13. Das TP, Suman S and Damodaran C: Induction of reactive oxygen species generation inhibits epithelial-mesenchymal transition and promotes growth arrest in prostate cancer cells. Mol Carcinog 53: 537-547, 2014

14. Cho KH, Choi MJ, Jeong KJ, Kim JJ, Hwang MH, Shin SC, Park CG and Lee HY: A ROS/STAT3/HIF-1 $\alpha$ signaling cascade mediates EGF-induced TWIST1 expression and prostate cancer cell invasion. Prostate 74: 528-536, 2014.

15. Wu WS, Wu JR and Hu CT: Signal cross talks for sustained MAPK activation and cell migration: The potential role of reactive oxygen species. Cancer Metastasis Rev 27: 303-314, 2008.

16. Livak KJ and Schmittgen TD: Analysis of relative gene expression data using real-time quantitative PCR and the 2(-Delta Delta C(T)) method. Methods 25: 402-408, 2001.

17. Li F, Wang H, Huang C, Lin J, Zhu G, Hu R and Feng H: Hydrogen peroxide contributes to the manganese superoxide dismutase promotion of migration and invasion in glioma cells. Free Radic Res 45: 1154-1161, 2011.

18. Hsieh HL, Wang HH, Wu WB, Chu PJ and Yang CM: Transforming growth factor- $\beta 1$ induces matrix metalloproteinase- 9 and cell migration in astrocytes: Roles of ROS-dependent ERK- and JNK-NF- $\mathrm{B}$ B pathways. J Neuroinflammation 7: 88, 2010.

19. Wu Y and Zhou BP: New insights of epithelial-mesenchymal transition in cancer metastasis. Acta Biochim Biophys Sin (Shanghai) 40: 643-650, 2008

20. Wray CJ, Ahmad SA, Matthews JB and Lowy AM: Surgery for pancreatic cancer: Recent controversies and current practice. Gastroenterology 128: 1626-1641, 2005.

21. Iwatsuki M, Mimori K, Yokobori T, Ishi H, Beppu T, Nakamori S, Baba $\mathrm{H}$ and Mori M: Epithelial-mesenchymal transition in cancer development and its clinical significance. Cancer Sci 101: 293-299, 2010.

22. Chiu WT, Shen SC, Chow JM, Lin CW, Shia LT and Chen YC: Contribution of reactive oxygen species to migration/invasion of human glioblastoma cells U87 via ERK-dependent COX-2/ PGE(2) activation. Neurobiol Dis 37: 118-129, 2010.

23. Chaiswing L, Zhong W, Cullen JJ, Oberley LW and Oberley TD: Extracellular redox state regulates features associated with prostate cancer cell invasion. Cancer Res 68: 5820-5826, 2008.

24. Hempel N, Carrico PM and Melendez JA: Manganese superoxide dismutase (Sod2) and redox-control of signaling events that drive metastasis. Anticancer Agents Med Chem 11: 191-201, 2011.

25. Chen PM, Wu TC, Wang YC, Cheng YW, Sheu GT, Chen CY and Lee $\mathrm{H}$ : Activation of NF- $\kappa \mathrm{B}$ by SOD2 promotes the aggressiveness of lung adenocarcinoma by modulating NKX2-1-mediated IKK $\beta$ expression. Carcinogenesis 34: 2655-2663, 2013.

26. Kinnula VL and Crapo JD: Superoxide dismutases in malignant cells and human tumors. Free Radic Biol Med 36: 718-744, 2004.

27. Toh Y, Kuninaka S, Oshiro T, Ikeda Y, Nakashima H, Baba H, Kohnoe S, Okamura T, Mori M and Sugimachi K: Overexpression of manganese superoxide dismutase mRNA may correlate with aggressiveness in gastric and colorectal adenocarcinomas. Int J Oncol 17: 107-112, 2000.

28. Wang S, Wang F, Shi X, Dai J, Peng Y, Guo X, Wang X, Shen $\mathrm{H}$ and $\mathrm{Hu} \mathrm{Z}$ : Association between manganese superoxide dismutase (MnSOD) Val-9Ala polymorphism and cancer risk A meta-analysis. Eur J Cancer 45: 2874-2881, 2009.

29. Tang H, Dong X, Day RS, Hassan MM and Li D: Antioxidant genes, diabetes and dietary antioxidants in association with risk of pancreatic cancer. Carcinogenesis 31: 607-613, 2010. 
30. Connor KM, Hempel N, Nelson KK, Dabiri G, Gamarra A Belarmino J, Van De Water L, Mian BM and Melendez JA: Manganese superoxide dismutase enhances the invasive and migratory activity of tumor cells. Cancer Res 67: 10260-10267, 2007.

31. Mori K, Shibanuma M and Nose K: Invasive potential induced under long-term oxidative stress in mammary epithelial cells. Cancer Res 64: 7464-7472, 2004.

32. Kim MC, Cui FJ and Kim Y: Hydrogen peroxide promotes epithelial to mesenchymal transition and stemness in human malignant mesothelioma cells. Asian Pac J Cancer Prev 14: 3625-3630, 2013.
33. Lee KH, Kim SW and Kim JR: Reactive oxygen species regulate urokinase plasminogen activator expression and cell invasion via mitogen-activated protein kinase pathways after treatment with hepatocyte growth factor in stomach cancer cells. J Exp Clin Cancer Res 28: 73, 2009.

34. Liu J, Ben QW, Yao WY, Zhang JJ, Chen DF, He XY, Li L and Yuan YZ: BMP2 induces PANC-1 cell invasion by MMP-2 overexpression through ROS and ERK. Front Biosci (Landmark Ed) 17: 2541-2549, 2012

35. Gilmore TD: Introduction to NF-kappaB: Players, pathways, perspectives. Oncogene 25: 6680-6684, 2006. 